\section{Alkohol in Phytopharmaka: ist das nicht riskant?}

\author{
Sind pflanzliche Medikamente immer so harmlos, wie viele Ärzte \\ und Patienten glauben? Können Zusätze und Konservierungsstoffe \\ auf Alkoholbasis für Kinder oder alte Menschen gefährlich werden? \\ Prof. A.-M. Beer, Hattingen, nimmt Stellung.
}

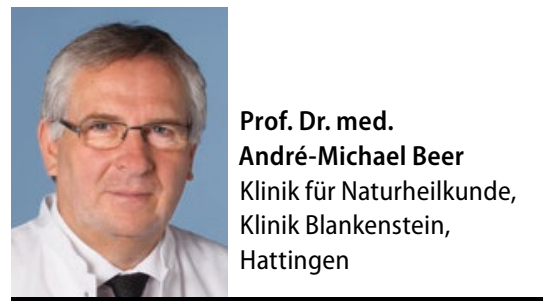

eingenommen wird. Bei Alkoholkranken ist bei der Verordnung alkoholhaltiger Medikamente allerdings stets Vorsicht geboten.

Praxisrelevant ist die Alkoholfrage bei Kindern, die dem Wunsch der Eltern nach unbedenklichen Arzneimitteln entsprechend häufig Phytopharmaka in flüssigen Darreichungen wie Tropfen, Frischpflanzensäfte, Sirupe oder Teeaufgüssen erhalten. Formale und legislative Probleme bestehen in der dürftigen Datenlage zum Alkoholgehalt in flüssigen Zubereitungen. Viele synthetische und pflanzliche Arzneimittel werden in der Pädiatrie nach OffLabel-Use angewandt.

Da Phytopharmaka bei akuten, schweren, lebensbedrohlichen Krankheiten nicht eingesetzt werden und über eine große therapeutische Breite verfügen, erübrigt sich die Berechnung nach Körperoberfläche und Gewicht; als Faustregel hat sich das Alter bewährt: 1.-2. Lebensjahr ein Viertel, 3.-6. Lebensjahr ein Drittel, 6.-9. Lebensjahr die Hälfte und 10.-16. Lebensjahr zwei Drittel der Erwachsenendosis.

Was den Alkoholgehalt in flüssigen Darreichungen angeht, so ist darauf hinzuweisen, dass Apfel- und Orangensaft ca. $0,4 \%$ Ethanol enthalten und ein 12-Jähriger nach Genuss von 0,2 1 ca. $0,64 \mathrm{~g}$ Ethanol aufnimmt, woraus ein Blutalkoholspiegel von 0,036\%o resultiert. In der EMA-Richtlinie werden maximal 1,5 g Ethanol/Dosis für 6-Jährige maximal 2,8 g Ethanol/Dosis für 12-Jährige und für Kinder unter zwei Jahren keine alkoholhaltigen Darreichungsformen empfohlen [1].

\section{? Wie ist die Situation bei alten Patienten?}

In der Altersmedizin ist auf die Galenik zu achten. Als galenische Zubereitungsart bieten sich in der Geriatrie die Frischpflanzenpresssäfte an. Es handelt sich um eine alkoholfreie galenische Form, die gegenüber den Medizinalkräutertees reicher an pharmakologisch aktiven Inhaltsstoffen ist. In der Regel besitzen Frischpflanzenpresssäfte daher eine höhere Wirksamkeit im Vergleich zu den Medizinalkräutertees und können ärztlich verordnet werden. Als weitere alkoholfreie galenische Zubereitung kommt für alte Patienten eine Vielzahl von Sirupen in Frage. In der Geriatrie haben sich Sirupe mit dem Eibisch, der Süßholzwurzel etc. neben ca. 20 anderen Sirupen bewährt.

Aus organoleptischen Gründen kommen in der Geriatrie die Medizinalweine in Frage. Arzneiweine auf der Basis von Xereswein mit einem durchschnittlichen Alkoholgehalt von ca. 15\% Vol. sind in verschiedenen Arzneibüchern offizinell und werden von älteren Patienten gerne als „Stomachicum“ eingenommen. In Frage kommt vor allem China-, Condurango-, Calamus- und Pepsinwein.

\section{Literatur}

1. EMA. Reflection paper on ethanol content in herbal medicinal products used in children. EMA/HMPC/85114/2008

Weitere Infos

Dossier Naturheilkunde

$\rightarrow$ www.springermedizin.de/239812 